\title{
Clinico-Mycological Study of dermatophytosis in a Tertiary Care Hospital
}

\author{
Rejitha Kurukkanari¹, Girija Kalarikkal Rajagopal2², Vimalraj Angattukuzhiyil Narayanan³ ${ }^{3}$ Neelakandhan Asokan ${ }^{4}$ \\ ${ }^{1}$ Department of Microbiology, Government Medical College, Manjeri, Malappuram, Kerala, India. ${ }^{2}$ Department of \\ Microbiology, Amritha Institute of Medical Sciences, Kochi, Kerala, India. ${ }^{3}$ Department of Microbiology, Government Medical \\ College, Thrissur, Kerala, India. ${ }^{4}$ Department of Dermatology, Government Medical College, Thrissur, Kerala, India.
}

\section{ABSTRACT}

\section{BACKGROUND}

Dermatophytes are fungi that infects the skin, hair and nails. They are hyaline septate moulds with more than hundred species described. Of these, 42 species are considered as valid and less than half are associated with human diseases. Dermatophytoses are infections produced by these and are common in tropical and subtropical areas of the country with high humidity. ${ }^{1}$ We wanted to speciate dermatophytes using phenotypic methods, analyze the risk factors, and study their clinical correlation.

\section{METHODS}

The study was conducted in a tertiary care hospital in South India over a period of one year. All newly suspected cases of dermatophytosis attending Dermatology Outpatient Department were selected for the study. Thus, a total of 113 patients were enrolled in the study. Samples from these patients were subjected to direct microscopy and culture was done on Sabouraud Dextrose Agar with antibiotics. Potato dextrose agar was used for enhancement of pigment production. Culture confirmation and speciation were done by tease mount, slide culture and supplemental tests like urease test and hair perforation test.

\section{RESULTS}

The present study was carried out on 113 clinically diagnosed cases of dermatophytoses. Maximum number of cases occurred in the 11-20 years age group and slight female preponderance was noted. Tinea corporis was the most common type of dermatophytosis, 68 cases $(60.2 \%)$ followed by mixed type (tinea corporis + tinea cruris) 14 cases $(12.4 \%)$ and tinea cruris 13 cases $(11.5 \%)$. Overall positivity by culture was $39 \%$ and by direct microscopy $96 \%$. Trichophyton rubrum was the most predominant species - 18 isolates (38.3\%) and most of them were isolated from tinea corporis. Trichophyton verrucosum (25.5\%) and Trichophyton mentagrophyte $(21.3 \%)$ were also obtained as major isolates.

\section{CONCLUSIONS}

This study highlighted that tinea corporis is the commonest clinical type. Trichophyton rubrum is the most predominant species. But we got a fairly good number of Trichophyton verrucosum and Trichophyton mentagrophyte also. Exposure to predisposing factors were present in almost half of the cases. Dermatophytosis is a trivial disease and antifungal agents are the drugs of choice for treatment but identification of predisposing factors and avoidance of these can decrease the incidence of the disease to some extent.

\section{KEY WORDS}

Dermatophytosis, Dermatophytes, Tinea, Trichophyton, Lactophenol Cotton Blue (LPCB)
Corresponding Author: Rejitha Kurukkanari, Aaramam, Padikkunnu, P. O. Nilambur, Malappuram-679329, Kerala, India.

E-mail: rejithak.2037@gmail.com

DOI: 10.14260/jemds/2020/46

Financial or Other Competing Interests: None.

How to Cite This Article: Kurukkanari R, Rajagopal GK, Narayanan $V A$, et al. Clinico-mycological study of dermatophytosis in a tertiary care hospital. J. Evolution Med. Dent. Sci. 2020;9(04):195-199, DOI: 10.14260/jemds/2020/46

Submission 16-11-2019,

Peer Review 08-01-2020,

Acceptance 13-01-2020,

Published 27-01-2020. 


\section{BACKGROUND}

Dermatophytes are filamentous fungi which infect the keratinized layers of skin, hair and nails. They are more prevalent in tropical and sub-tropical areas². These fungi belong to three main anamorphic genera: Epidermophyton, Microsporum and Trichophyton. Disease produced by these fungi is known as dermatophytosis, characterized by dermal inflammatory response with severe itching. It is also known as 'ring worm' infection or 'tinea'. The name ringworm comes from the worm like appearance of the lesions with irregular inflammatory borders. Tinea is a Latin word for "ring worm". Infection is named according to the body site after the word tinea. ${ }^{1}$ The prevalent species of dermatophyte varies considerably in different geographical areas of the country.2,3 Based on their ecological characteristics, dermatophytes are divided into geophilic, zoophilic and anthropophilic species. Heat and moisture play an important role in promoting the growth of these Fungi.4 Other factors such as Diabetes mellitus, immunocompromised states, use of steroids, poor hygiene, exposure to cattle rearing areas, taking bath in untidy ponds and dams, overcrowding etc. also promote increased incidence. $4,5,6,7,8$ The first scientific proof of the dermatophytosis was provided by Remak in 1845 . Since then many have surveyed and isolated the dermatophytes in different countries.6,7,8,9 In India Dr. Powell first reported the case of dermatophytosis from Upper Assam ${ }^{3}$ in 1900.

The infection is common in coastal areas like Kerala and remains a public health problem. So far, not much data is available about the pattern of the disease in and around the central part of Kerala. Hence this study was being undertaken to know the disease pattern in patients attending the Government medical College, Thrissur.

\section{METHODS}

This study was conducted in a tertiary care center in South India over a period of one year after getting approval by the Institutional Ethics Committee. A total of 113 clinically suspected cases of dermatophytoses attending Dermatology Outpatient Department were taken for the study. All age groups of both sexes were included in the study. Samples were taken after getting written consent from the patient or from the parent in case of children. A detailed history including age, sex, occupation, duration, and site of infection were taken. Patients who were already on treatment were excluded from the study.

\section{Specimen Collection}

Lesions were cleaned with $70 \%$ alcohol to remove the dirt and contaminating bacteria. In case of skin lesions active border of the lesion was selected and scrapings were taken with the blunt end of a sterilized scalpel. In affected nails, clippings along with the subungual debris were collected with a nail cutter. Hairs were collected by plucking them with the forceps so that the roots were preserved intact. Samples were collected in a sterile paper - folded, labelled and then transported to microbiology lab at room temperature and no specific transport media used. Further processing was carried out under mycology section. Materials were divided into two parts one for wet mount examination $(10 \% \mathrm{KOH}$ for skin lesions and $40 \% \mathrm{KOH}$ for nail and hair) and the other for culture. Wet mount $(\mathrm{KOH})$ : In a positive preparation the fungi appeared as septate branching hyphal elements, highly refractile among the epidermal scales.

\section{Culture}

Specimens were inoculated into Sabouraud's Dextrose Agar (Accumix) with antibiotics - chloramphenicol $(50 \mathrm{mg} / \mathrm{L})$ and cycloheximide $(500 \mathrm{mg} / \mathrm{L})$. The tubes were incubated at room temperature for 4 weeks. If there was no growth after 4 weeks the culture was considered as negative. In culture positive cases, colony morphology was recorded weekly including topography (flat, raised, folded or cerebriform), texture (granular, velvety or cottony) and pigmentation (obverse or reverse, diffusible or non-diffusible). After observing the colony morphology, they were speciated by the standard procedures. ${ }^{1,2,10}$ Lactophenol cotton blue mount (LPCB) were taken from the culture to study the morphology of the hyphae (Septate or not, its diameter), conidia and their arrangement. Slide culture was done for those isolates that could not be identified by LPCB mount. The slides were examined under low power and then under high power. The arrangement of hyphae and conidia were noted. Adhesive cellophane tape preparation done for those isolates which were plated for better sporulation. Aerial hyphae adhere to the tape and examined by placing on a drop of LPCB on a slide. Microscopic examination done for the presence of conidia i.e. microconidia and macroconidia - its number, size, shape and thickness were noted. In case of macroconidia number of cells or compartments were also recorded. Presence of accessory structures such as chlamydospores and pectinate hyphae were also observed. Species were identified by urease test to differentiate between Trichophyton rubrum (urease negative) and Trichophyton mentagrophyte (urease positive) and hair perforation test to differentiate between Trichophyton mentagrophyte and Trichophyton rubrum. Wedge shaped perforation was formed for Trichophyton mentagrophyte whereas the absence of perforation was typical for Trichophyton rubrum. Pigment production by Dermatophyte species was enhanced (e.g.: red pigment by trichophyton rubrum) by growing them on Potato dextrose agar and cornmeal agar (HiMedia) with 1\% dextrose. Growth in Lowenstein- Jensen medium (HiMedia) was noted for Trichophyton soudanense.

\section{Statistical Analysis}

The analyzed results were expressed as percentage for the description of the patterns of distribution of various clinical types of dermatophytoses and various species of dermatophytes. Association between the clinical types of dermatophytoses and the species isolated were also studied. Chi square distribution was used to test the qualitative distribution. Predictive value ( $\mathrm{p}$ value) of less than 0.05 was considered as a significant association between the variables tested.

\section{RESULTS}

The present study for isolation, identification and clinicomycological study of dermatophytes was done on 113 
clinically diagnosed cases of dermatophytosis. Out of these 113 samples: 94 (83.1\%) were skin scrapings, 7 (6.2\%) were nail clippings and $2(1.77 \%)$ were hair stubs. Out of 113 clinically diagnosed cases of dermatophytoses, males accounted for $48.7 \%$ (55 cases) and females 51.3\% (58 cases). The male female ratio was $0.95: 1$ The maximum number of cases were found in the age group of 11-20 yrs. 29 cases $(25.7 \%)$ followed by $31-40$ yrs. 21 cases (18.6\% ) [see table 1]

\begin{tabular}{|c|c|c|}
\hline Age Group (Yrs.) & Number of Cases & Percentage (\%) \\
\hline 0 to 10 & 14 & 12.4 \\
\hline $11-20$ & 29 & 25.7 \\
\hline $21-30$ & 15 & 13.3 \\
\hline $31-40$ & 21 & 18.6 \\
\hline $41-50$ & 18 & 15.9 \\
\hline $51-60$ & 7 & 6.2 \\
\hline $61-70$ & 8 & 7.1 \\
\hline $71-80$ & 1 & 1.1 \\
\hline \multicolumn{3}{|c|}{ Table 1. Distribution of Patients with } \\
Dermatophytoses According to Age \\
\hline \multicolumn{3}{|c}{} \\
\hline
\end{tabular}

Of the total 113 patients, 57 cases (50.3\%) had no exposure to predisposing factors whereas $56(49.7 \%)$ had history of some predisposing factors. 32 (28.3) cases had history of using occlusive dressings, $10(8.8 \%)$ had history of taking bath in ponds/dams, $6(5.3 \%)$ had contact with affected family members, 5 cases $(4.4 \%)$ had contact with pet animals and $4(3.5 \%)$ cases had cattle rearing in house [see table 2].

\begin{tabular}{|c|c|c|}
\hline Predisposing Factors & Number & $\mathbf{\%}$ \\
\hline No exposure & 57 & 50.3 \\
\hline Occlusive dressings & 32 & 28.3 \\
\hline Frequent bath in stagnant waters - (Ponds \& dams) & 10 & 8.8 \\
\hline Affected family members & 6 & 5.3 \\
\hline Contact with dog/cat & 5 & 4.4 \\
\hline Cattle rearing in house & 4 & 3.5 \\
\hline Total & $\mathbf{1 1 3}$ & $\mathbf{1 0 0}$ \\
\hline Table 2. Predisposing Factors of Dermatophytoses \\
\hline
\end{tabular}

Tinea corporis was found to be the commonest clinical type with 68 cases $(60.2 \%)$ followed by mixed type (Tinea corporis + tinea cruris) 14 (12.4\%), tinea cruris $13(11.5 \%)$, tinea unguium 7 (6.2\%), tinea pedis $4(3.5 \%)$, tinea faciei 4 $(3.5 \%)$, tinea capitis $2(1.8 \%)$ and tinea manuum $1(0.9 \%)$. [see table 3]

\begin{tabular}{|c|c|c|c|}
\hline Sl. No. & Clinical Types & No. of Cases & Percentage (\%) \\
\hline 1 & Tinea corporis & 68 & 60.2 \\
\hline 2 & Tinea corporis+ Tinea cruris & 14 & 12.4 \\
\hline 3 & Tinea cruris & 13 & 11.5 \\
\hline 4 & Tinea unguium & 7 & 6.2 \\
\hline 5 & Tinea pedis & 4 & 3.5 \\
\hline 6 & Tinea faciei & 4 & 3.5 \\
\hline 7 & Tinea capitis & 2 & 1.8 \\
\hline 8 & Tinea manuum & 1 & 0.9 \\
\hline \multicolumn{3}{|c|}{ Table 3. Clinical Types of Dermatophytoses } \\
\hline
\end{tabular}

Out of 113 cases of dermatophytoses, 96 (85\%) cases were positive by direct microscopy and 17 (15\%) were negative. Among the 96 wet mount positive cases only 43 were culture positives, remaining 53 did not grow in culture. Of the 17 wet mount negative cases 4 samples yielded dermatophyte isolates. Remaining 13 cases were negative by direct smear as well as culture. Sensitivity of microscopy was $91.5 \%$ and specificity was $19.7 \%$.

Of the total 113 cases 47 (41.6\%) were culture positive as mentioned above. Trichophyton rubrum was the commonest isolate 18 (38.3\%). Other isolates were Trichophyton verrucosum 12 (25.5\%), Trichophyton mentagrophyte 10(21.3\%), Trichophyton tonsurans 3 (6.4\%), Trichophyton soudanense 2 (4.3\%) and Microsporum gypseum 2 (see table 4)

\begin{tabular}{|c|c|c|}
\hline Species & Number of isolates & Percentage (\%) \\
\hline T. rubrum & 18 & 38.3 \\
\hline T. verrucosum & 12 & 25.5 \\
\hline T. mentagrophytes & 10 & 21.3 \\
\hline T. tonsurans & 3 & 6.4 \\
\hline T. soudanense & 2 & 4.3 \\
\hline M. gypseum & 2 & 4.3 \\
\hline \multicolumn{2}{|c|}{ Table 4. Species of Dermatophytes Isolated } \\
\hline
\end{tabular}

Of the total 18 isolates of Trichophyton rubrum 10 $(55.6 \%)$ were from tinea corporis, $3(16.7 \%)$ from tinea cruris, 2 (11.1\%) from mixed type and 1 (5.6\%) from tinea pedis, tinea faciei and tinea unguium each. Trichophyton verrucosum was the second common type i.e., 12 isolates (25.5\%) 8 from tinea corporis, 3 from mixed type and 1 from tinea cruris. Trichophyton mentagrophytes were 10 (21.3\%). Of these $5(50 \%)$ were from tinea corporis, $3(30 \%)$ from mixed type and $1(10 \%)$ from tinea cruris and tinea faciei each. Trichophyton tonsurans was the fourth type 3 isolates $6.4 \%, 1$ from tinea corporis and 2 from tinea unguium lesions. $2(4.3 \%)$ isolates of Trichophyton soudanense obtained from 2 cases of tinea cruris and Microsporum gypseum obtained from 2 (4.3\%) cases, 1 from tinea corporis and tinea capitis each. (see table 5)

\begin{tabular}{|c|c|c|c|c|c|c|c|}
\hline & $\begin{array}{c}\mathbf{5} \\
\mathfrak{5} \\
\mathfrak{5} \\
\mathfrak{5}\end{array}$ & 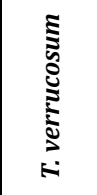 & 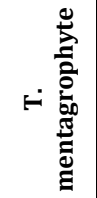 & 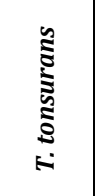 & 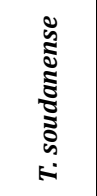 & 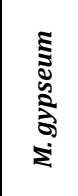 & $\begin{array}{l}\text { 표 } \\
\stackrel{0}{ }\end{array}$ \\
\hline Tinea corporis & $10(55.6 \%)$ & $8(66.7 \%)$ & $5(50.0 \%)$ & $1(33.3 \%)$ & - & $1(50 \%)$ & 25 \\
\hline Tinea cruris & $3(16.7 \%)$ & $1(8.3 \%)$ & $1(10 \%)$ & - & $2(100 \%)$ & - & 7 \\
\hline T. corporis +T. cruris & $2(11.1 \%)$ & $3(25.0 \%)$ & $3(30 \%)$ & - & - & - & 8 \\
\hline Tinea pedis & $1(5.6 \%)$ & - & - & - & - & - & 1 \\
\hline Tinea manuum & & - & - & - & - & - & 0 \\
\hline Tinea faciei & $1(5.6 \%)$ & - & $1(10 \%)$ & - & - & - & 2 \\
\hline Tinea capitis & - & - & - & - & - & $1(50 \%)$ & 1 \\
\hline Tinea unguium & $1(5.6 \%)$ & - & - & $2(66.7 \%)$ & - & - & 3 \\
\hline Total & 18 & 12 & 10 & 3 & 2 & 2 & 47 \\
\hline Percentage & $38.3 \%$ & $25.5 \%$ & $21.3 \%$ & $6.4 \%$ & $4.3 \%$ & $4.3 \%$ & $100 \%$ \\
\hline Table 5 & & of $\mathrm{Va}$ & Is $S p$ & $\begin{array}{l}\text { es of } D \\
\text { ypes }\end{array}$ & nat & tes in & \\
\hline
\end{tabular}

Association was tested by Chi square test. $\mathrm{P}$ value calculated as 0.002 , indicating that there is significant association between clinical types and species isolated.

\section{DISCUSSION}

Studies on dermatophytoses in India have received much attention in recent years because of the increasing incidence of the mycotic infections worldwide. Dermatophytoses can occur at any age. In the present study the occurrence of disease was more in the age group of $10-20$ years $(25.7 \%)$. Vineetha $\mathrm{Met}^{9}{ }^{9}$ in 2018 reported maximum number of cases of dermatophytoses in the age group of 10-20 years. But various other workers reported maximum number of cases in 20-30 years of age group. ${ }^{7,9,11,12}$ The higher incidence in 
young age may be due to increased physical activity, use of cosmetics, prolonged studying pattern and hormonal pattern. In our study 21 cases (18.6\%) occurred in 30-40 years and most of them were housewives. This was due to their involvement in household activities most of the times. In the present study incidence of Infection was almost equal in both sexes with a slight preponderance in females. M: F ratio was 0.95:1.

Vineetha $\mathrm{M}^{\text {et }} \mathrm{al}^{9}$ in 2018 conducted a mycological study on dermatophytoses in Kottayam and found that the incidence was more in females in the first episode with a male female ratio 1:1.1. But studies conducted by KAK Surendran et al in 2011 and Hosthota A et al in 2018 revealed male preponderance with the male female ratio of $1.63: 1$ and 2.06:1 respectively. Of the total 113 cases, 56 had history of predisposing factors such as occlusive dressing pattern, use of cosmetics and continuous studying pattern which may create the favourable environment such as increased moisture or exposure to cattle rearing areas where chance of getting zoophilic dermatophytes is high.5,9,11,13 68.3\% of patients belonged to middle class. This was in accordance with the study by Agarwal US ${ }^{7}$ in 2014 in which $60 \%$ belonged to middle class. But various other studies revealed that the incidence was more in low socio-economic groups. $4,5,14$ This change is attributed to changing lifestyles mainly.

Out of 113 cases, 96 cases (85\%) were positive on $\mathrm{KOH}$ mount. Similar higher positivity was reported in the study of Agarwal US7 (84.67\%). Culture positivity was observed in 47 cases $(41.6 \%)$. Overall positivity was $40 \%$ in the study by Noronha et al. Out of 47 positives, $43(91.5 \%)$ were positive by $\mathrm{KOH} \&$ remaining $4(8.5 \%)$ were negative. In the study by $\mathrm{K}$ a K Surendran, out of the total 39 culture positives, 35 $(89.7 \%)$ were $\mathrm{KOH}$ positive and the remaining $4(10.3 \%)$ were $\mathrm{KOH}$ negative. Sensitivity of microscopy was $91.5 \%$. Specificity of microscopy was $19.7 \%$.

Predictive value of positive microscopy was $44.8 \%$. Predictive value of negative microscopy was $76.5 \%$. Among the 47 dermatophyte species isolated Trichophyton rubrum was the most common agent 18 (38.3\%) causing dermatophytosis. Other workers who reported Trichophyton rubrum as predominant isolate in their study were KAK Surendran in 2014 (67.5\%), Sumit Kumar ${ }^{8}$ in 2014 (65.09\%), Agarwal US7 in 2014, Vineetha $\mathrm{M}^{9}$ in 2018 and Abhineetha Hosthota in 2018.

The common occurrence of Trichophyton rubrum in various parts of the country may be due to its greater adaptability to survive in varying climatic conditions and warm and humid climate as in central part of Kerala. Next common were Trichophyton verrucosum 12 (25.5\%) and Trichophyton mentagrophyte 10 (21.3\%). Higher number of isolates of Trichophyton verrucosum was also reported by Belurkar DD (21.6\%) and Noronha T M (8.3\%). In this study we got a fairly good number of Trichophyton verrucosum species. An important fact is that $3.5 \%$ of patients in our study were having cattle rearing in houses and $8.8 \%$ were having the habit of taking bath in common places such as ponds, dams etc. where the herd animals frequently come in contact.

Aghamirian MR and Ghiasian S A ET al $^{5}$ conducted an epidemiological study of dermatophytes as a cause of epizoonoses in dairy cattle and humans in Iran during 2006-
2007. It revealed that $33.1 \%$ of herdsmen developed fungal lesions suspected of dermatophytoses which on further examination, $\mathrm{KOH}$ and culture isolated Trichophyton verrucosum in $62.8 \%$, similar to the isolate obtained from the affected cattle (92.6\%). Trichophyton mentagrophyte was the second commonest isolate by Surendran et al (20\%), Kumar et $\mathrm{al}^{8}(17.92 \%)$, and Hosthota $\mathrm{A}$ et al $(20 \%)$ in 2018 . In the present study Trichophyton tonsurans isolates were $3(6.4 \%)$ of the total 47 isolates. Of these 1 (33.3\%) was from tinea corporis and $2(66.7 \%)$ from tinea unguium. Trichophyton tonsurans was the common isolate from tinea unguium in the study by BelurkarDD. ${ }^{4}$

Trichophyton soudanense species was obtained from 2 cases $(4.3 \%)$ in this study and they were siblings, one was a 10 -year-old boy and the other 7-year-old girl. Both were having tinea cruris type of lesions. Trichophyton soudanense is a common species causing tinea capitis ${ }^{1}$. But there were no tinea capitis lesions when these patients came. According to the literature ${ }^{2}$ it can involve other parts of the body also. Microsporum gypseum was obtained from 2 cases (4.3\%) in the present study. One from tinea corporis and the other from tinea capitis. Microsporum gypseum is a common isolate causing tinea capitis mainly kerion type. Similar findings were obtained by Sumana V. ${ }^{6}$ In the present study the commonest isolate was Trichophyton rubrum 18 (38.3\%) from all clinical isolates.

In tinea corporis 10 isolates (55.6\%), in tinea cruris 3 isolates $(16.7 \%)$, and in mixed type of infection (tinea corporis + tinea cruris) 2 isolates were Trichophyton rubrum from culture. 1 case of tinea pedis, tinea faciei and tinea unguium also yielded Trichophyton rubrum. Other studies also supported the present pattern. In the study done by Sumit Kumar and Srikara Mallya in 2014 commonest isolate was Trichophyton rubrum 69 (65.09\%). ${ }^{3}$ In tinea corporis 34 isolates $(61.82 \%)$, in tinea cruris 26 isolates $(74.28 \%)$, in tinea unguium 3 isolates $(60 \%)$, in tinea pedis 2 isolates $(100 \%)$ in tinea capitis 1 isolate $(20 \%)$ and in tinea manuum 1 isolate (50\%) were Trichophyton rubrum. In tinea faciei, tinea pedis and in tinea pedis and only Trichophyton rubrum was isolated. In the study done by Hosthota $\mathrm{A}$ et al ${ }^{7}$ in 2018 Trichophyton rubrum was the main isolate in all clinical types.

Similar observation was found in the study by $\mathrm{K}$ a $\mathrm{K}$ Surendran ET al ${ }^{11}$ and various other workers. ${ }^{9}$ In the present study tinea corporis was the predominant type accounting for 68 cases $(60.2 \%)$ followed by the mixed type (Tinea corporis + tinea cruris - 14 cases, $12.4 \%$ ) and tinea cruris 13 (11.5\%) cases, almost equal incidence. Kumar ET al ${ }^{8}$ in 2014 and Vineetha $\mathrm{M}$ et $\mathrm{al}^{9}$ in 2018 observed tinea corporis as the commonest clinical type $(47.6 \%)$ and $(28.7 \%)$ respectively. KAK Surendran ${ }^{11}$ reported higher incidence of tinea corporis followed by tinea cruris. Huda $\mathrm{MM}^{3}$ et al reported that mixed type was the most common type. Tinea cruris was the most common type $(50 \%)$ in the study by Hosthota A.

Tinea unguium was found in 7 cases $(6.2 \%)$ in this study. KAK Surendran also found similar occurrence pattern (8.1\%). The incidence is more in females in our series. There were 4 $(3.5 \%)$ cases of tinea pedis and tinea faciei. Similar lower incidence was reported by $\mathrm{K}$ a $\mathrm{K}$ Surendran (tinea pedis $22.7 \%$ and tinea faciei $1.3 \%$ ). All the tinea pedis cases were in females. There were 2 cases of tinea capitis and both of these 
were boys less than 10 yrs. of age. Both lesions were of kerion type. Many authors reported higher incidence of tinea capitis in children.6,8,15 Tinea manuum was noted in $1(1.1 \%)$ elderly male patient. Similar lower incidence was reported by K A K Surendran (3.3\%). No tinea manuum cases were reported by Hosthota A et al.

\section{CONCLUSIONS}

Tinea corporis is the most common clinical type and Trichophyton rubrum is the most common causative agent. Unlike other studies we obtained a fairly good number of Trichophyton verrucosum and Trichophyton mentagrophyte species as well. This is probably due to exposure to cattle rearing areas and habits of taking bath in ponds and dams in this part of Thrissur, besides other common predisposing factors. Antifungal agents are the drugs of choice for dermatophytosis. But identification of predisposing factors and avoidance of these can prevent the occurrence of the disease to some extent.

\section{ACKNOWLEDGEMENT}

Authors thank the staff of Department of Dermatology and Department of Microbiology.

\section{REFERENCES}

[1] Rippon JW. Medical mycology. $3^{\text {rd }}$ edn. Philadelphia: W B Saunders Company, 1988: p. 169-275.

[2] Padhey AA, Summerbell RC. Medical mycology - Topley and Wilson's Microbiology and microbial infections. $10^{\text {th }}$ edn. Oxford University, London: 1998: p. 223-32.

[3] Huda MM, Chakroborty N, Bordoloi JNS. A clinicmycological study of superficial mycoses in Upper Assam. Indian J Dermatol Venereol Leprol 1995;61(6):329-32.
[4] Belurkar DD, Bharma RN, Kartikeyan S, et al. A mycological study of dermatophytoses in Thane. Indian J Pathol Microbiol 2003;21:329-36.

[5] Aghamirian MR, Ghiasian SA. Dermatophytoses as a cause of epizoonoses in dairy cattle and humans in Iran: epidemiological and clinical aspects. Mycoses 2011;54(4):e52-6.

[6] Sumana V, Singaracharya MA. Dermatophytoses in Khammam (Khammam District, Andhra Pradesh, India). Indian J Pathol Microbiol 2004;47(2):287-9.

[7] Agarwal US, Saran J, Agarwal P. Clinico-mycological study of dermatophytes in a tertiary care center in North West India. Indian J Dermatol Venereol Leprol 2014;80(2):194.

[8] Kumar S, Mallya PS, Kumari P. Clinico- mycological study of dermatophytoses in a tertiary care hospital. International Journal of Scientific Study 2014; 1 (6):2732.

[9] Vineetha M, Sheeja S, Celine MI, et al. Profile of dermatophysis in a tertiary care center. Indian Journal of Dermatology 2018; 63 (6):490-5.

[10] Walsh TH, Hayden RT, Larone DH. Larone's medically important fungi- a guide to identification. $6^{\text {th }}$ edn. Washington, DC: Wiley Blackwell, ASM books, 2018: p. 249-362.

[11] Surendran KAK, Bhat RM, Boloor R, et al. A clinical and mycological study of dermatophytic infection. Indian J Dermatol Venereol Leprol 2014; 59 (3) :262-7.

[12] Hosthota A, Gowda T, Manikanda R. Clinical profile and risk factors of dermatophytoses: a hospital based study. International Journal of Research in Dermatology 2018; $4(4): 508-13$

[13] Verma S, Madhu R. The great Indian epidemic of superficial dermatophytoses. Indian Journal Dermatol 2017; 62 (3):227-36.

[14] Noronha TM, Tophakhane RS, Nadiger S. Cliniccomicrobiological study of dermatophytosis in a tertiary care hospital in North Karnataka. Indian Dermatol Online Journal 2016; 7 (4):264-71.

[15] Mishra N, Rastogi MK, Gahalaut P, et al. Clinicomycological study of dermatophytoses in children: presenting at a tertiary care center. Indian journal of Paediatric Dermatology 2018; 19 (4):326-30. 